\title{
AVALIAÇÃO QUÍMICA DE LENHOS CARBONIZADOS DE Araucaria columnaris SOB DIFERENTES CONCENTRAÇÕES DE OXIGÊNIO COMO COMPARATIVO DE ANÁLISE DE charcoal FÓSSIL
}

\author{
Fernanda Mardera, Daniela Mueller de Laraa,b, André Jasper ${ }^{\mathrm{c}}$, Eduardo Miranda Ethur ${ }^{\mathrm{a}}$, Dieter Uhld e Simone Stülp ${ }^{\mathrm{a}, *,(\bullet)}$ \\ ${ }^{a}$ Centro de Ciências Exatas e Tecnológicas, Universidade do Vale do Taquari, 95914-014 Lajeado - RS, Brasil \\ bUniversidade Estadual do Rio Grande do Sul, Unidade do Alto da Serra Botucaraí Soledade, 99300-000, Soledade - RS, Brasil \\ ${ }^{c}$ Centro de Ciências Biológicas e da Saúde, Universidade do Vale do Taquari, 95900-000 Lajeado - RS, Brasil \\ dSenckenberg Forschungsinstitut und Naturmuseum, 60325, Frankfurt am Main, Germany/Senckenberg Center for Human Evolution \\ and Palaeoenvironment, Institut für Geowissenschaften, Universität Tübingen, 72076, Tübingen, Germany
}

Recebido em 03/08/2020; aceito em 03/11/2020; publicado na web em 10/12/2020

\begin{abstract}
CHEMICAL EVALUATION OF Araucaria columnaris CARBONIZED WOODS UNDER DIVERSE OXYGEN CONCENTRATIONS AS COMPARATIVE ANALYSIS OF FOSSIL charcoal. In the face of environmental changes concerns, the study of climate change becomes essential to understand the life trajectory of planet earth. For this purpose, this work seeks to understand climate change and fluctuations in the amount of atmospheric oxygen across geological ages, carbonizing the Araucaria columnaris wood in varying oxygen concentrations, and comparing them to the fossil coal from Quiteria Outcrop. In this study, pyrolysis of Araucaria columnaris wood occurred with the use of TGA, under an atmosphere of $21 \%$ and $30 \% \mathrm{O}_{2}$, analyzed in FTIR, distinguishing the constituent compounds of the wood. Multivariate analysis of the mains components (PCA) was applied, for data crossing. There is a distinction between carbonized wood and fossil charcoal, the charcoal burning temperature as well as the atmospheric oxygen composition being inconclusive. However, FTIR results indicate that the amount of oxygen has an influence on the degradation of the wood, and samples at $450{ }^{\circ} \mathrm{C}$ have greater similarities with fresh wood. Thus, it was observed that the temperature and the burning time are the main factors of the fires, being that the amount of oxygen available in the atmosphere influences the firing process.
\end{abstract}

Keywords: charcoal; atmospheric oxygen; thermogravimetric analysis; charred wood.

\section{INTRODUÇÃO}

O fogo é um processo ecológico chave que afeta a dinâmica da vegetação e a cobertura da terra, ${ }^{1}$ desde que as plantas terrestres evoluíram há 420 milhões de anos, e tem desempenhado um papel na formação da composição e fisionomia de muitos ecossistemas desde então. ${ }^{2} \mathrm{O}$ charcoal, um detrito lenhoso, é um dos poucos legados duradouros dos incêndios florestais ${ }^{3}$ que fornece informações do registro de atividades culturais, condições climáticas passadas e de um meio de controle cronológico de vida. ${ }^{4}$ Ainda, há uma necessidade urgente de documentar e entender sua ocorrência no passado geológico, sendo que o charcoal é um dos mecanismos para entender a história da Terra, por ser um indicador paleoambiental, paleoclimático e geodinâmico inestimável, devido a registros detalhados e de longo prazo que ele contém. As características do charcoal podem ser interpretadas pelo estudo de sua composição botânica, geoquímica, mineralógica, palinológica e petrológica. ${ }^{5}$

A ocorrência dos paleoincêndios está ligada a fatores ambientais, como concentração de oxigênio atmosférico, disponibilidade de combustível e fonte de ignição. ${ }^{6-8}$ Sendo que a interpretação de tais eventos fornece informações significativas paleoambientais. ${ }^{8}$

Ao longo dos tempos geológicos, flutuações nos níveis de oxigênio atmosférico influenciaram a evolução biológica e tiveram um papel integral nos mecanismos de feedback que regem os ciclos biogeoquímicos da Terra. ${ }^{6}$ Ao qual afetaram de forma drástica os regimes de incêndios no passado, e as quantidades de carvão fóssil variavam em função dos níveis atmosféricos estimados de $\mathrm{O}_{2}{ }^{2}$

$\mathrm{O} \mathrm{O}_{2}$ atmosférico mostra um pico largo no período neopaleozoico com valor máximo de $30 \%$ de $\mathrm{O}_{2}$ no Permiano, um valor menor

*e-mail: stulp@univates.br perto do Siluriano limite/Devonianos, variação entre $15 \%$ e $20 \%$ de $\mathrm{O}_{2}$ durante o Cambriano e Ordovícico, uma queda muito acentuada de $30 \%$ a $15 \%$ de $\mathrm{O}_{2}$ atmosférico no limite Permo-Triássico, e um aumento mais ou menos contínuo de $\mathrm{O}_{2}$ desde o Triássico Tardio até o presente. ${ }^{9}$

Através dos períodos em que o $\mathrm{O}_{2}$ se apresentou em quantidade elevada na atmosfera, como no Permo-Carbonífero, acredita-se que o percentual do $\mathrm{O}_{2}$ atmosférico tenha atingido, provavelmente, níveis $>30 \%$. Com isso, os incêndios não foram só generalizados e frequentes, mas intensos e presentes nas copas das árvores. ${ }^{2}$

O estudo das características morfológicas do lenho após a combustão pode fornecer algum insight sobre a intensidade do fogo no passado ou o tipo de material vegetal queimado, ${ }^{10,11}$ no entanto, estimativas da disposição de oxigênio na atmosfera no momento dos incêndios passados não são comumente feitos.

Além disso, os indicadores representativos e qualitativos do paleoclima são mecanismos que fornecem evidências de mudanças climáticas em grande escala, que ocorreram antes dos registros documentais instrumentais. A técnica de uso de testemunho (do inglês proxy, "representante", "substituto") a qual se baseia em informações biológicas, geológicas e geofísicas, possibilita o estudo para reconstruir estimativas bastante próximas das condições climáticas reais ocorridas no passado, inclusive aquelas precedentes aos alguns milhares de anos da história humana. ${ }^{12,13}$

Neste estudo foi selecionada a espécie da família Araucariaceae por serem gimnospermas do gênero das coníferas, assemelhando-se a espécies de fósseis catalogadas, iniciando no Carbonífero, Cenozoico, Jurássico, Neogene, Permiano, Paleogene e Triássico. ${ }^{14}$ Cabe salientar que as primeiras ocorrências de charcoal no Afloramento Quitéria ${ }^{15}$ para o Paleozoico Superior da Bacia do Paraná, foram estudadas por Jasper ${ }^{16}$ em 2006, e confirmadas em estudos posteriores. ${ }^{17-21}$ 
O Afloramento corresponde a uma sequência deposicional de $8 \mathrm{~m}$ de espessura, que se intercala desde um argilito carbonoso até paraconglomerados com matriz sílica e arenosa, como camadas de charcoal de até $25 \mathrm{~cm}$ de espessura, contendo afinidades taxonômicas que confirmam a sua correlação com coníferas e licófitas, elementos comuns no nível de roof-shale. ${ }^{20}$ Estudos relacionados a flutuações climáticas possibilitam identificar causas e mecanismos de variações no clima ao longo de milhares de anos. Com o auxílio de modelos climáticos, a capacidade de reproduzir coerentemente situações do paleoclima é fundamental para simular, de forma robusta, a fim de prever antecipadamente as mudanças que possam ocorrer no futuro. ${ }^{22}$

Dessa forma, a complexa relação entre o fogo e o planeta terra está diretamente relacionada com a evolução dos biomas. E, diante do cenário de preocupações com as mudanças ambientais que o mundo vem demonstrando, o estudo de mudanças climáticas se torna essencial para entendermos a trajetória de vida da Terra. Trabalhos sobre os paleoincêndios são uma das ferramentas que podem ser utilizadas para compreender as mudanças que a atmosfera passou ao longo dos milhões de anos.

Nesse contexto, o objetivo do presente trabalho é avaliar as alterações que ocorrem durante o processo de carbonização dos lenhos da família Araucariaceae in natura em diferentes condições atmosféricas, com o intuito de ampliar conhecimentos acerca do período em que ocorreram as formações de charcoal fóssil e posteriormente comparar quimicamente com amostras encontradas em campo, do Período Permiano.

\section{PARTE EXPERIMENTAL}

O estudo desenvolvido possui natureza aplicada, avaliando as alterações físicas e químicas que ocorrem durante o processo de carbonização de lenho da família Araucariaceae in natura, em exposição a diferentes atmosferas gasosas controladas em laboratório e comparadas com charcoal (Período Permiano).

\section{Localização e coleta da espécie de Araucaria columnaris}

Para a realização deste estudo foi coletada no município de

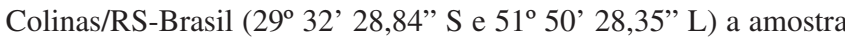
de Araucária conforme a legislação ambiental vigente. $\mathrm{O}$ fragmento de lenho foi retirado na forma de um disco completo de $8,0 \mathrm{~cm}$ de espessura diretamente de um tronco de Araucaria columnaris no momento da sua derrubada. Seguindo padrões de levantamentos de dados fitossociológicos, o material foi retirado a uma altura entre 130,0 e 150,0 cm em relação ao nível do solo, o que corresponde ao Diâmetro à Altura do Peito (DAP) que variou entre 18,0 e 35,0 cm. O exemplar seco não recebeu nenhum tratamento químico-físico e foi acondicionado em laboratório, sendo posteriormente utilizado in natura. Para a identificação das amostras a nomenclatura utilizada é a in natura (amostra antes do processo de queima), amostras carbonizadas (após o processo de queima artificial) e charcoal (carvão vegetal macroscópico).

\section{Caracterização dos lenhos in natura por análise termogravimétrica}

As amostras de lenho da Araucaria columnaris (Figura 1) foram carbonizadas no analisador termogravimétrico Perkin Elmer, modelo TGA-4000, a fim de simular condições atmosféricas variadas. As análises foram realizadas em quintuplicata, a $20 \mathrm{mg}( \pm 2 \mathrm{mg})$ sob atmosfera de $21 \% \mathrm{O}_{2}$ e $30 \% \mathrm{O}_{2}$, a vazão constante de $20 \mathrm{~mL} \mathrm{~min}^{-1} \mathrm{e}$ temperatura ambiente (em torno de $25^{\circ} \mathrm{C}$ ), com taxa de aquecimento a $25^{\circ} \mathrm{C} \mathrm{min}^{-1}$.

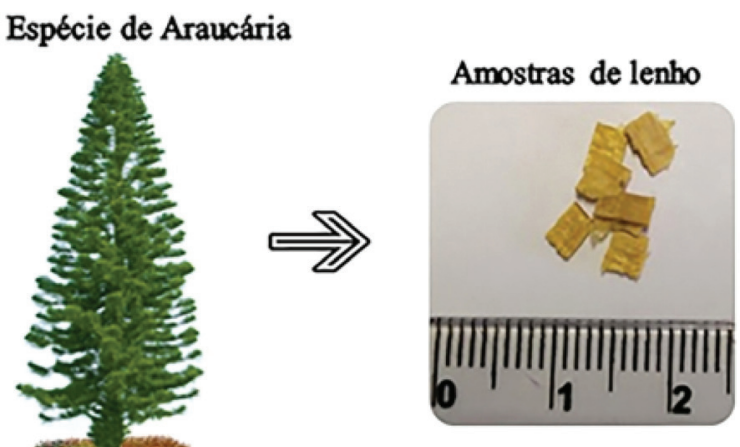

Figura 1. Amostragem do lenho de Araucariaceae

A pirólise dos lenhos ocorreu em atmosfera com $21 \%$ e $30 \% \mathrm{O}_{2}$ (em ensaios distintos), com aquecimento na faixa de $25^{\circ} \mathrm{C}$ a $450{ }^{\circ} \mathrm{C}$, e $25^{\circ} \mathrm{C}$ a $600{ }^{\circ} \mathrm{C}$. Após esse procedimento, duplicatas das amostras foram submetidas à análise termogravimétrica sob atmosfera de $\mathrm{N}_{2}$, incluindo uma amostra in natura (lenho que não possui nenhuma forma de tratamento), de $25{ }^{\circ} \mathrm{C}$ a $600{ }^{\circ} \mathrm{C}$, vazão constante de $20 \mathrm{~mL} \mathrm{~min}^{-1}$ e temperatura ambiente (em torno de $25^{\circ} \mathrm{C}$ ).

A massa residual durante o processo carbonização foi calculada a partir da massa inicial de cada amostra subtraindo-se o valor da massa final obtida e expressas em porcentagem.

O estudo da quantidade de oxigênio na carbonização dos lenhos se deve à estimativa do percentual atmosférico encontrado no Período Permiano, em torno de $30 \% \mathrm{O}_{2}$, conforme estudos. ${ }^{2}$ Sendo também os charcoal do Afloramento Quitéria, que datam desse período. Já os $21 \%$ de $\mathrm{O}_{2}$ referem-se à composição atmosférica atual, podendo-se, assim, realizar a comparação das amostras com as simulações das queimadas nos dias de hoje.

\section{Localização do Afloramento Quitéria}

As amostras de charcoal foram coletadas no Afloramento Quitéria (Figura 2), localizado no município de Encruzilhada do Sul porção centro-oeste do Estado do Rio Grande do Sul/Brasil, na borda sudeste da Bacia do Paraná, limitado no cruzamento das coordenadas geográficas $30^{\circ} 17^{\prime} \mathrm{S}-52^{\circ} 11^{\prime} \mathrm{W}$.

As amostras analisadas são provenientes do Afloramento Quitéria (Figura 2) e estão depositados na coleção Paleobotânica do Museu de Ciências da Univates, onde estão tombadas sob o acrônimo PbUMCN (614 e 624). Coletadas em uma sequência de intervalo entre 0,9-4,2 $\mathrm{cm}$ de comprimento e $0,3-0,7 \mathrm{~cm}$ de espessura e possuindo afinidades taxonômicas que confirmam a sua correlação com coníferas e licófitas, elementos comuns no nível de roof-shale.

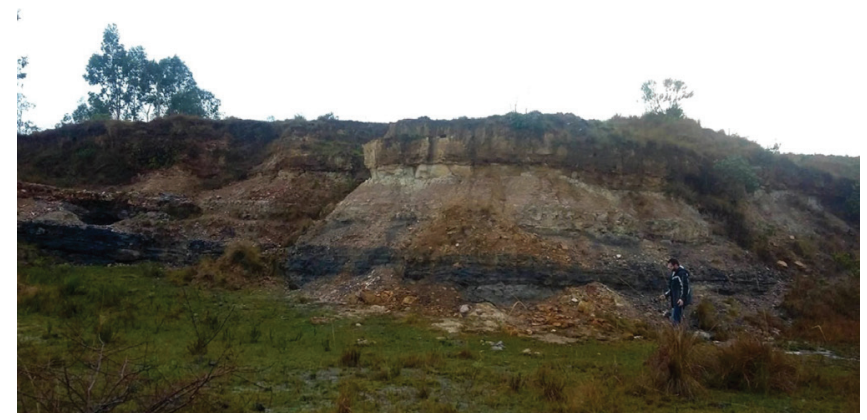

Figura 2. Afloramento Quitéria

\section{Análise infravermelha dos lenhos}

Após a carbonização do lenho em atmosfera de $\mathrm{O}_{2}$, as amostras 
foram lidas em espectrômetro de infravermelho com transformada de Fourier (FTIR), SHIMADZU, modelo IR Affinity-1, em triplicatas. Amostras de lenhos in natura e carbonizados foram preparados conforme o método de confecção de pastilhas de $\mathrm{KBr}$ (brometo de potássio). ${ }^{23} \mathrm{Na}$ leitura do branco (background) e das amostras foi utilizado um intervalo entre 4000 a $400 \mathrm{~cm}^{-1}$, com resolução de $4 \mathrm{~cm}^{-1}$ e 64 varreduras.

Para a aquisição dos espectros no infravermelho médio foi realizado primeiramente a leitura do branco (background). Os espectros para cada amostra foram adquiridos em triplicata, sendo que os gráficos avaliados foram os espectros médios de cada condição analisada. Ainda, a técnica de FTIR foi utilizada para analisar amostras de charcoal fóssil.

\section{Análise multivariada}

A Análise de Componentes Principais (PCA) foi realizada com o software ChemoStat. ${ }^{24}$ Os dados dos espectros de FTIR obtidos, entre o intervalo entre 4000 a $400 \mathrm{~cm}^{-1}$, foram previamente suavizados pelo método de Savitsky-Golay, centrados na média e aplicado a primeira derivada antes de serem submetidos à PCA.

A PCA foi realizada utilizando-se um total de 18 amostras (média das 3 replicatas por temperatura avaliada, por espécie analisada e por charcoal fóssil).

\section{RESULTADOS E DISCUSSÃO}

Acompanhar as mudanças na química do charcoal ao longo do tempo tem o potencial de revelar a temperatura de combustão de incêndios passados e, consequentemente, fornecer insights sobre seus impactos ecológicos. ${ }^{10}$

A Figura 3 exibe os dados das análises termogravimétricas do charcoal fóssil e do lenho in natura e carbonizado (em diferentes atmosferas de $\mathrm{O}_{2}$ e temperaturas de $450{ }^{\circ} \mathrm{C}$ e $600{ }^{\circ} \mathrm{C}$ ), obtidos em atmosfera de nitrogênio com taxa de aquecimento de $25{ }^{\circ} \mathrm{C}$ $\min ^{-1}$, a fim de verificar as particularidades da queima do lenho de A. columnaris. ${ }^{25}$

As temperaturas de incêndios ocorrentes no solo superficial em florestas temperadas geralmente variam entre $400{ }^{\circ} \mathrm{C}$ e $600{ }^{\circ} \mathrm{C}$, embora as condições diretamente em dejetos lenhosos grosseiros ardentes possam exceder $700{ }^{\circ} \mathrm{C} .{ }^{3}$ Devido a essa leitura, nos estudos de Lara, ${ }^{26}$ os melhores resultados foram obtidos em temperaturas de $450{ }^{\circ} \mathrm{C}$ e $600{ }^{\circ} \mathrm{C}$, sendo o motivo de escolha para a pirólise do lenho neste estudo.

A biomassa encontrada junto ao lenho consiste em alta massa molecular de hemicelulose, celulose, lignina, e componentes de baixa massa, como extrativos e as cinzas. ${ }^{27}$ Sendo que esses elementos cobrem respectivamente $20-40 \%$ (hemicelulose), $40-60 \%$ (celulose) e $10-25 \%$ (lignina) em peso da biomassa lignocelulósica. ${ }^{28,29}$

Em relação ao processo de carbonização, esse resulta na degradação estrutural da madeira (hemicelulose, celulose e lignina), enquanto a água, $\mathrm{CO}, \mathrm{CO}_{2}$ e outros gases são libertados. ${ }^{7,13}$

As termogravimetrias apresentadas na Figura 3 fornecem características térmicas (sob atmosfera de $\mathrm{N}_{2}$ ) dos lenhos e charcoal avaliados neste estudo. Com o aumento da temperatura, observam, inicialmente, as mudanças no lenho em termos morfológicos, o escurecimento da madeira; físicos - considerável perda de massa, retração e possível distorção anatômica do material original resultante da formação e perda de muitos voláteis; e químicos - a conversão contínua e gradual da celulose, hemicelulose e lignina em porções predominantemente aromáticas, formando assim um novo produto final quimicamente distinto e enriquecido em carbono. ${ }^{30}$

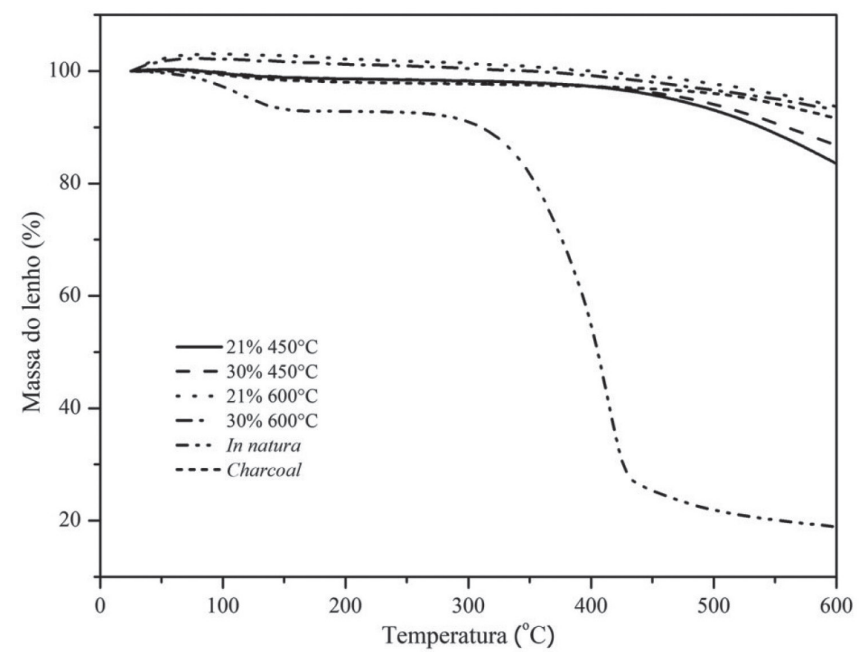

Figura 3. Análises termogravimétricas de charcoal e do lenho de A. columnaris in natura e carbonizado (em diferentes atmosferas de $\mathrm{O}_{2}$ e temperaturas de $450{ }^{\circ} \mathrm{C}$ e $600^{\circ} \mathrm{C}$ ), obtidos em atmosfera de nitrogênio e taxa de aquecimento de $25^{\circ} \mathrm{C}$ min $^{-1}$

Comparando as curvas de degradação do material, verificouse que há uma maior estabilização térmica das amostras com a queima em temperaturas superiores, em $600{ }^{\circ} \mathrm{C}$. Os charcoal fósseis apresentam características semelhante às faixas de degradação térmica das amostras de lenho a $450{ }^{\circ} \mathrm{C}$, se tornando estáveis termicamente nos últimos $200^{\circ} \mathrm{C}$. Com a degradação da lignina gerou um aumento da resistência térmica e a estabilidade da amostra. Essas observações também foram realizadas nos estudos de Padilla, ${ }^{25}$ no qual espigas de milho carbonizadas em maior temperatura apresentaram as mesmas características do material exposto ao nitrogênio.

$\mathrm{Na}$ Figura 3, observa-se que um aumento no $\mathrm{O}_{2}$ facilita a ignição do combustível, ${ }^{2}$ aumentando a taxa de combustão, levando a um maior fluxo de energia pela queima de combustíveis. $\mathrm{O}$ processo exotérmico tem início entre $270^{\circ} \mathrm{C}$ a $290^{\circ} \mathrm{C}$, no qual o lenho continua seu processo de aquecimento, entre $290^{\circ} \mathrm{C}$ a $400^{\circ} \mathrm{C}$ há a liberação de gases combustíveis, como dióxido de carbono, monóxido de carbono, ácido acético e metanol e vapores condensáveis. A transformação é praticamente completa quando a temperatura ultrapassa $400{ }^{\circ} \mathrm{C} . .^{31}$

A hemicelulose tem sua decomposição na faixa de $220{ }^{\circ} \mathrm{C}$ a $315^{\circ} \mathrm{C}$, podendo ser observada no lenho carbonizado. Na Figura 3, as maiores temperaturas tiveram maior estabilidade do lenho e resistência térmica. ${ }^{25}$

A pirólise da celulose ocorre numa faixa maior de temperatura, entre 260 e $370{ }^{\circ} \mathrm{C}$. Sendo que acima de $370{ }^{\circ} \mathrm{C}$, a celulose se decompõe quase que por completo em um intervalo de tempo curto. ${ }^{32}$

Observa-se que o estudo realizado apresenta similaridade com os resultados descritos por Padilha, ${ }^{25}$ comparando-se as curvas de degradação, a estabilidade térmica dos lenhos carbonizados e do charcoal fóssil aumentaram com a temperatura de queima e refletindo diretamente nas alterações da estrutura química.

Os resultados de DTG (termogravimetria derivada) nos fornecem informações da degradação do lenho carbonizado e do charcoal fóssil. Observa-se na Figura 4 que as modificações químicas significativas ocorreram no lenho in natura com o aumento da temperatura, com perda de $81,07 \%$ de biomassa. Os lenhos carbonizados e o charcoal fóssil não sofreram uma variação significativa na pirólise em $\mathrm{N}_{2}$, sendo possível que a queima dos constituintes da biomassa tenha ocorrido na carbonização do lenho na presença de $\mathrm{O}_{2}$.

Observa-se que charcoal fóssil apresenta semelhanças de degradação térmica ao lenho carbonizado a $450{ }^{\circ} \mathrm{C}$, sendo que possivelmente foi submetido a temperaturas semelhantes no incêndio 


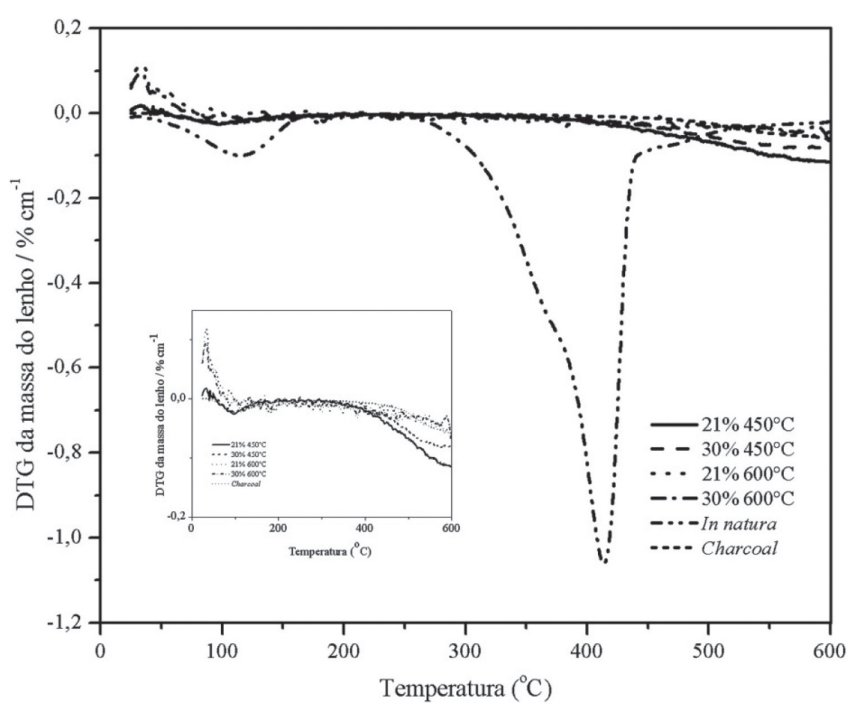

Figura 4. Curvas DTG para o charcoal e o lenho carbonizado, obtidos em atmosfera de nitrogênio e taxa de aquecimento de $25^{\circ} \mathrm{C} \mathrm{min}{ }^{-1}$. Gráfico contendo tratamento de 50 pontos de Smooth para melhor análise

florestal. No entanto, o processo nos mostra que há decomposição da lignina a partir do $400{ }^{\circ} \mathrm{C}$, tornando a liberação de energia com a perda de massa mais intensa, aproximando a amostra aos lenhos carbonizados a $600{ }^{\circ} \mathrm{C} . .^{33,34}$

Verifica-se na Figura 4, que houve uma significativa redução de biomassa durante o processo de carbonização, sendo que em $30 \%$ de $\mathrm{O}_{2}$ e $600{ }^{\circ} \mathrm{C}$, degradou-se $87,16 \%$ do lenho, e em $21 \%$ de $\mathrm{O}_{2}$, perdeu-se $85,17 \%$. Já em atmosfera de $21 \%$ de $\mathrm{O}_{2}$ à $450{ }^{\circ} \mathrm{C}$, a perda foi menor, $71,82 \%$ da biomassa e a $30 \%$ de $\mathrm{O}_{2}, 65,14 \%$. Segundo Figueroa e Moraes, ${ }^{35}$ a perda da biomassa alcança mais de $80 \%, 95 \%$ e $60 \%$ a $600{ }^{\circ} \mathrm{C}$ para a celulose, a hemicelulose e a lignina respectivamente, comprovando pela degradação das amostras estudadas. A degradação pode ser compreendida por meio de quatro estágios individuais: ${ }^{25}$ evolução da umidade, decomposição da hemicelulose, decomposição da celulose e decomposição da lignina.

Na Figura 5 pode-se observar o espectro de absorção na região infravermelha, com amostras de lenho carbonizadas em atmosferas de $21 \%$ e $30 \%$ de $\mathrm{O}_{2}$, além do lenho in natura.

A espectroscopia do infravermelho foi utilizada para investigar diferenças no carvão em termos de informações sobre a temperatura de combustão e as mudanças químicas após a pirólise. ${ }^{10,25}$

As amostras após tratamento em atmosfera contendo $30 \%$ de $\mathrm{O}_{2}$, apresentaram uma maior degradação quando submetidas a $600{ }^{\circ} \mathrm{C}$, em que há maior incidência de oxigênio na queima e consequentemente maior impacto na degradação em comparação com a atmosfera à $21 \%$ de $\mathrm{O}_{2}$.

No gráfico apresentado na Figura 5 foram identificados os principais grupos funcionais e respectivas regiões de absorção. Comparando as duas atmosferas de $\mathrm{O}_{2}$ estudadas, observa-se que houve uma maior degradação do lenho na atmosfera de $30 \%$ de $\mathrm{O}_{2}$, sendo que a atmosfera de $21 \%$ de $\mathrm{O}_{2}$ se assemelha ao lenho in natura. Isso ocorreu devido à degeneração da hemicelulose, lignina e celulose da biomassa. Em comparação com lenho in natura, foram identificados os principais grupos funcionais e respectivas regiões de absorção: ${ }^{23,25,28,36,37}$ a deformação axial do grupo $\mathrm{OH}\left(3452 \mathrm{~cm}^{-1}\right)$ associada à ligação de hidrogênio aos grupos funcionais de fenóis, álcoois, presente na cadeia molecular da celulose, hemicelulose e lignina; deformação axial alifática do $\mathrm{CH}\left(2903 \mathrm{~cm}^{-1}\right)$ ligações C-H de grupo $\mathrm{CH}_{3}, \mathrm{CH}_{2}$ e $\mathrm{CH}$ de alcanos e de grupo alquinos; alongamento de $\mathrm{C}=\mathrm{O}\left(1750 \mathrm{~cm}^{-1}\right)$, associada à cetona, presente na hemicelulose; $\mathrm{C}=\mathrm{C}$ alongamento de grupos aromáticos, benzeno $\left(1646 \mathrm{~cm}^{-1}\right)$;

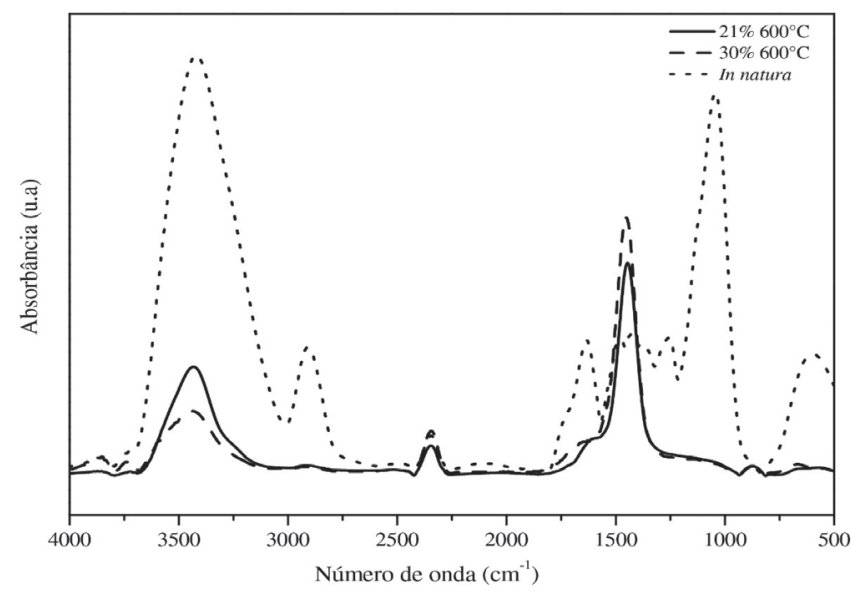

Figura 5. Espectros de FTIR do lenho carbonizado e in natura, a $21 \%$ e $30 \%$ de $\mathrm{O}_{2}$, a $600^{\circ} \mathrm{C}$. Gráfico contendo tratamento de 67 pontos de Smooth e linha de base para melhor análise

presença O-C-O $\left(1049 \mathrm{~cm}^{-1}\right)$, acetal (cuja intensidade está relacionada à quantidade de celulose).

Ainda, observa-se uma maior degradação nos picos referentes ao $\mathrm{OH}$ e ao $\mathrm{CH}_{n}$, devido à desidratação do lenho e da quebra de ligações químicas durante perda de biomassa no processo de queima. No entanto, a região na qual as amostras obtiveram uma maior variação de picos entre o lenho carbonizado e in natura foi entre $1250 \mathrm{a} 500 \mathrm{~cm}^{-1}$, devido à degradação da celulose, hemicelulose e lignina, semelhante ao trabalho descrito por Padilha. ${ }^{25}$ A Figura 6 apresenta o espectro de FTIR mostrando a comparação do lenho carbonizado em diferentes temperaturas $\left(450{ }^{\circ} \mathrm{C}\right.$ e $\left.600{ }^{\circ} \mathrm{C}\right)$ em atmosfera de $30 \%$ de $\mathrm{O}_{2}$.

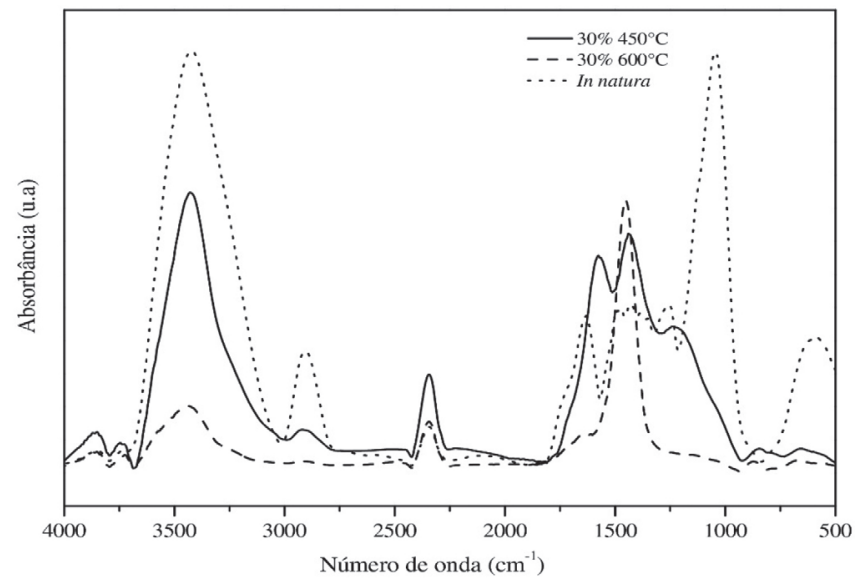

Figura 6. Espectros de FTIR do lenho carbonizado e in natura, à $30 \%$ de $\mathrm{O}_{2}$, nas temperaturas de $450^{\circ} \mathrm{C}$ e $600{ }^{\circ} \mathrm{C}$. Gráfico contendo tratamento de 67 pontos de Smooth e linha de base para melhor análise

Com base nos dados apresentados na Figura 6, observa-se que a perda da biomassa do lenho com queima à $600{ }^{\circ} \mathrm{C}$ foi maior que a ocorrida na temperatura de $450^{\circ} \mathrm{C}$, degradando as principais ligações químicas e refletindo na amostragem dos compostos presentes na composição do lenho.

Em relação à faixa espectral de 1900 a $650 \mathrm{~cm}^{-1}$, ao qual representa a impressão digital do composto, a degradação do lenho se torna visível. Isso ocorre devido à perda de voláteis da biomassa durante a pirólise, pois picos presentes nas amostras in natura e lenho carbonizado $\left(450{ }^{\circ} \mathrm{C}\right)$ não se encontram mais na amostra de $600{ }^{\circ} \mathrm{C}$

A perda de líquidos pode ser observada pela diminuição do alongamento $\mathrm{OH}\left(3452^{-1}\right)$, como também a quase inexistência do pico 
de $\mathrm{CH}\left(2903 \mathrm{~cm}^{-1}\right)$, devido à degradação dos compostos de celulose, hemicelulose e lignina. Essa, com menos degradação devido ao sistema aromático, composto por unidade de fenilpropano, presente em níveis mais elevados em coníferas e folhosas. ${ }^{33,38}$

$\mathrm{O}$ alongamento referente ao acetal, O-C-O $\left(1049 \mathrm{~cm}^{-1}\right)$ tem sua perda de biomassa quase que completa em comparação com o lenho in natura, devido à ligação ao composto de celulose, ao qual acima de $370{ }^{\circ} \mathrm{C}$ se decompõe quase por completo em um intervalo de tempo curto. ${ }^{32}$ Além disso, tem-se uma maior incidência de oxigênio na queima (concentração de $30 \%$ ), sendo, dessa maneira, maior a intensidade da degradação.

A PCA foi usada para focar a similaridade dos dados obtidos a partir das análises por FTIR. Considerando essa abordagem, o resultado da PCA demonstrou que com duas componentes principais é possível descrever 85,33\% dos dados (Figura 7).

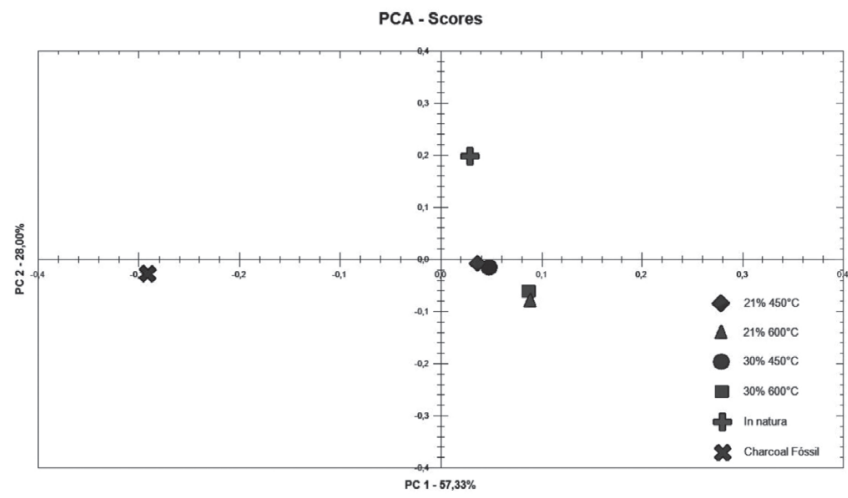

Figura 7. Representação dos scores para PC1 versus $P C 2$ das amostras de lenhos A. columnaris carbonizados em $450^{\circ} \mathrm{C}$ e $600^{\circ} \mathrm{C}$ em atmosferas de $21 \%$ e $30 \%$ de $\mathrm{O}_{2}$, Charcoal Fóssil e Lenhos in natura

A análise do gráfico dos scores (Figura 7) mostra as amostras separadas em diferentes agrupamentos. A PC1 separa as amostras para os lenhos de A. columnaris carbonizados artificialmente em $450{ }^{\circ} \mathrm{C}$ e $600{ }^{\circ} \mathrm{C}$ nas atmosferas de $21 \%$ e $30 \%$ de $\mathrm{O}_{2}$, e lenhos in natura em valores positivos ao longo do vetor, da amostra do charcoal fóssil, que possui valor negativo ao longo desta PC. Já a PC2 separa a amostra do charcoal e as amostras carbonizadas em $450{ }^{\circ} \mathrm{C}$ e $600{ }^{\circ} \mathrm{C}$ em atmosferas de $21 \%$ e $30 \%$ de $\mathrm{O}_{2}$ em valores negativos, do lenho in natura que apresenta valores positivos ao longo da PC. Essa separação se dá principalmente devido às variações na altura dos sinais de $\log (1 / \mathrm{R})$ situados próximos a 3600 a $3500 \mathrm{~cm}^{-1}$ e 1700 a $1400 \mathrm{~cm}^{-1}$ e 1200 a $900 \mathrm{~cm}^{-1}$.

Isso se torna mais evidente ao analisar o gráfico dos loadings referentes à PC1 (Figura 8). Os maiores valores absolutos de log (1/R) para os loadings da PC1 localizam-se nas regiões espectrais próximas a 1600 a $1500 \mathrm{~cm}^{-1}, 1100$ a $950 \mathrm{~cm}^{-1}, 950 \mathrm{~cm}^{-1}, 450$ a $400 \mathrm{~cm}^{-1}$ e vem ao encontro dos dados apresentados na Figura 6.

Conforme já apresentado na Figura 4, a degradação dos lenhos em atmosfera controlada evidencia a perda de massa das amostras avaliadas e uma semelhança espectral do charcoal com os lenhos carbonizados na atmosfera em $\mathrm{O}_{2}$, no entanto, com o modelo multivariado não é possível inferir qual a temperatura de queima do charcoal. Observa-se ainda que, os pontos próximos entre os lenhos carbonizados artificialmente à $450{ }^{\circ} \mathrm{C}$ e $600{ }^{\circ} \mathrm{C}$ não diferencia qual a atmosfera utilizada em termos de percentual de oxigênio $(21 \%$ ou $30 \%$ ) pois estão muito próximas entre si, devendo conter informações espectrais semelhantes.

Estudos realizados por Lara, ${ }^{26}$ evidenciaram na PCA o agrupamento de 3 distintos agrupamentos no processo de termodegradação dos lenhos carbonizados em TGA com atmosfera inerte. A PC1 separou as amostras com temperaturas de carbonização abaixo de $250^{\circ} \mathrm{C}$ em valores positivos ao longo do vetor, das amostras cuja carbonização foi realizada acima de $300^{\circ} \mathrm{C}$, que possuem valores negativos ao longo desta PC. Segundo os autores, a separação se dá principalmente devido às variações na altura dos sinais de $\log (1 / \mathrm{R})$ situados próximos a $1713 \mathrm{~cm}^{-1}, 1522 \mathrm{~cm}^{-1}, 1171 \mathrm{~cm}^{-1}$ e $982 \mathrm{~cm}^{-1}$. Ainda evidenciam que distanciamento entre as amostras carbonizadas pode estar atrelada ao maior desvio observado para os valores de perda de massa.

Lara e colaboradores ${ }^{39}$ ainda ressaltam que as amostras de charcoal avaliadas em seu estudo apresentaram características de queima acima de $300{ }^{\circ} \mathrm{C}$ e que os resultados obtidos estavam em concordância com Schmidt ${ }^{40}$ e Bica. ${ }^{41}$ No entanto, os resultados apresentados neste estudo indicam que as temperaturas de queima para ambos os processos (sem ausência de oxigênio, por TGA e com presença restrita de oxigênio, em mufla) atingiram temperaturas superiores do que apresentado por Schmidt ${ }^{40}$. Todavia, segundo os autores, devem ser levados em consideração os parâmetros de pressão, temperatura e ausência ou presença restrita de oxigênio no processo de termodegradação. Então, considerando-se que a metodologia foi desenvolvida na ausência e na presença restrita de oxigênio, se a quantidade de oxigênio presente no paleoambiente for superior a este estudo, a temperatura de carbonização do lenho pode ser antecipada.

Diante dos resultados obtidos, verifica-se que foi possível, por meio da caracterização realizada, comparar o comportamento de lenhos da família Araucariaceae in natura e carbonizados, e estabelecer relações com amostras de charcoal encontradas em campo, do Período Permiano. Os resultados expressaram que a disposição de oxigênio na atmosfera e as temperaturas das queimadas influenciam na degradação do lenho carbonizado, o que contribui na busca de um maior entendimento das flutuações de oxigênio na atmosfera, sendo que estudos como este podem igualmente contribuir para o entendimento dos processos ambientais atuais e futuros, com base em ocorrências pretéritas.

\section{CONCLUSÕES}

De modo geral, no trabalho desenvolvido, conclui-se que os lenhos carbonizados apresentam alterações significativas na estrutura física e química, sendo ambos transformados em charcoal (lenho carbonizado), inferindo que, para o charcoal fóssil, houve a degradação do lenho, com a perda de massa durante os incêndios florestais

O lenho carbonizado a $450{ }^{\circ} \mathrm{C}$ possui maior semelhança química com os lenhos in natura, já a $600{ }^{\circ} \mathrm{C}$, houve maior degradação, conforme a definição dos gráficos de FTIR. A quantidade de oxigênio atmosférico mostrou influência no processo de pirólise do lenho, sendo que a $30 \%$ de $\mathrm{O}_{2}$ obteve-se maior perda de massa e, consequentemente, quebra de ligações químicas comparadas com a atmosfera à $21 \%$ de $\mathrm{O}_{2}$.

O charcoal fóssil e o lenho carbonizado não mostraram semelhanças na análise multivariada, sendo impossibilitado de inferir a temperatura de queima do charcoal fóssil, bem como a composição de oxigênio atmosférico. Fatores como temperatura das queimadas, pressão e presença/ausência restrita de oxigênio durante a termodegradação podem ter influenciado os resultados obtidos.

Sendo assim, esses resultados expressam que a disposição de oxigênio na atmosfera e as temperaturas das queimadas influenciam na degradação do lenho carbonizado, o que contribui na busca de um maior entendimento das flutuações de oxigênio na atmosfera. 


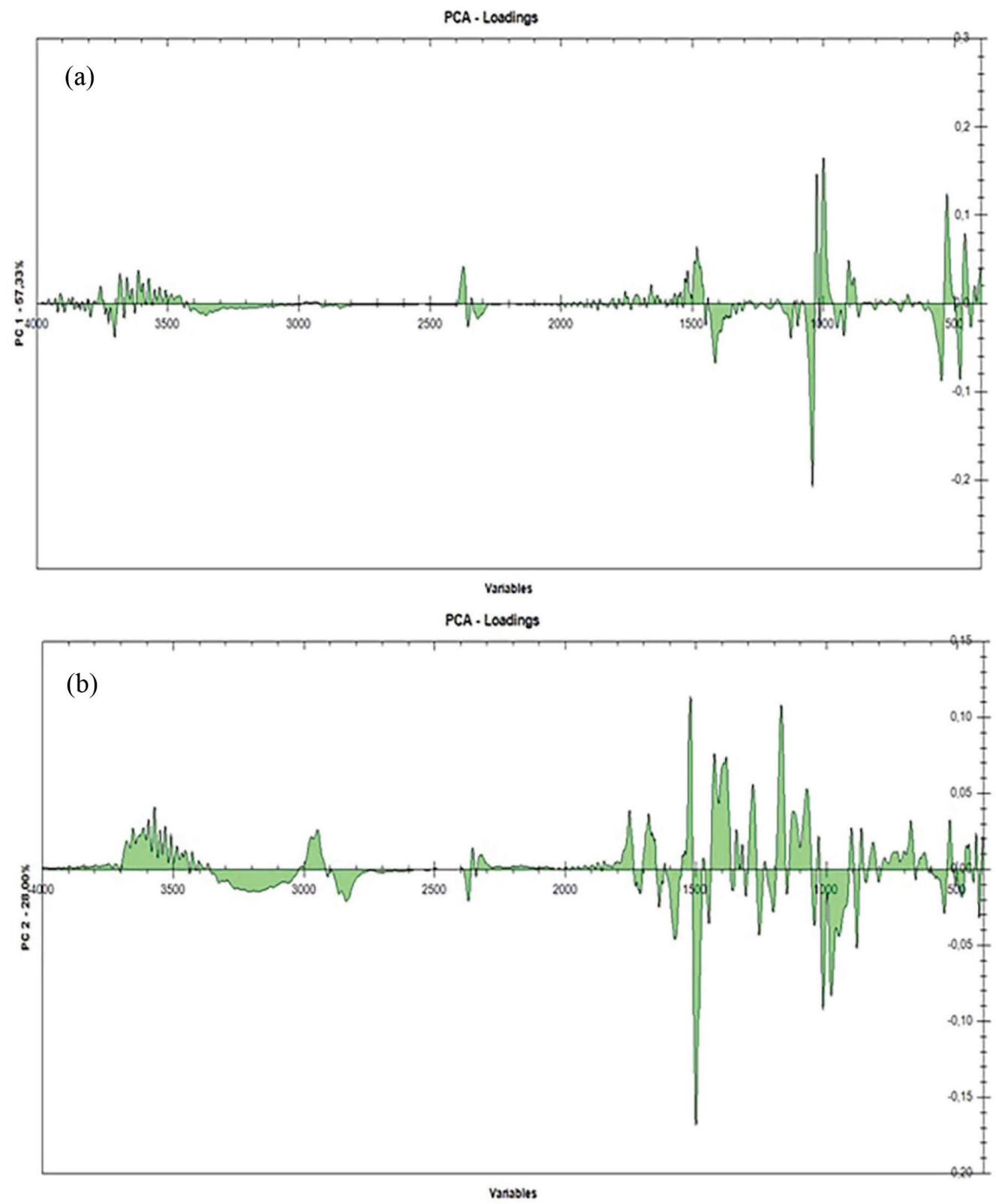

Figura 8. Loadings da componente principal 1 (a) e da componente principal 2 (b) para as amostras de lenhos de A. columnaris carbonizados à $450^{\circ} \mathrm{C} e$ $600{ }^{\circ} \mathrm{C}$ em atmosferas de $21 \%$ e $30 \%$ de $\mathrm{O}_{2}$

\section{AGRADECIMENTOS}

Os autores agradecem à Capes, ao $\mathrm{CNPq}\left(\mathrm{n}^{\circ}\right.$ processo 313694/2019-2 e 305938/2019-3), INCT Datrem, grupo de pesquisa do Núcleo de Eletrofotoquímica e Materiais Poliméricos (NEMP), ao Laboratório de Paleobotânica e Evolução de Biomas da Universidade do Vale do Traquari (UNIVATES) e ao Institut für Geowissenschaften, Universität Tübingen.

\section{REFERÊNCIAS}

1. Iglesias, V.; Yospin, G. I.; Whitlock, C.; Frontiers in Plant Science 2015, 5,785 .

2. He, T.; Lamont, B. B.; Natl. Sci. Rev. 2018, 5.

3. Pingree, M. R. A.; Deluca, E. E.; Schwartz, D. T.; Deluca, G.; Geoderma 2016, 283, 68.

4. Ascough, P. L.; Bird, M. I.; Francis, S. M.; Lebl, T.; J. Archaeol. Sci. 2011, 38, 69 .
5. Dai, S.; Bechtel, A.; Eble, C. F.; Flores, R. M.; French, D.; Graham, I. T.; Hood, M. M.; Hower, J. C.; Korasidis, V. A.; Moore, T. A.; Puttmann, W.; Wei, Q.; Zhao, L.; O’Keefe, J. M. K.; Int. J. Coal Geol. 2020, 219 , 1033832.

6. Scott. A. C.; Glasspool, I. J.; PENAS 2006, 103, 10861.

7. Scott, A. C.; Damblon, F.; Palaeogeogr. Palaeoclimatol. Palaeoecol. 2010, 291, 1 .

8. Manfroi, J.; Uhl, D.; Guerra-Sommer, M.; Francischini, H.; Martinelli, A. G.; Soares, M. B.; Jasper, A.; Palaeogeogr. Palaeoclimatol. Palaeoecol. 2015, 436, 77.

9. Berner, R. A.; Geochim. Cosmochim. Acta 2006, 70, 5653.

10. Gosling, W. D.; Cornelissen, H. L.; McMichael, C. N. H.; Palaeogeogr., Palaeoclimatol., Palaeoecol. 2019, 520, 128.

11. Umbanhowar Jr, C. E.; McGrath, M. J.; The Holocene 1998, 8, 3.

12. Oliveira, M. J.; Baptista, G. M. M.; Carneiro, C. D. R.; Vecchia, F. A. S.; Terra 2015, 12, 3 .

13. Ascough, P. L.; Bird, M. I.; Scott, A. C.; Collinson, M. E.; Cohen-Ofri, I.; Snape, C. E.; Manquais, K. L.; J. Archaeol. Sci. 2010, 37, 7. 
14. Renner, S. In The timetree of life; Hedges, S. B.; Kumar, S., orgs.; Oxford University Press: Oxford, 2009.

15. Jones, T. P.; Chaloner, W. G.; Paleo 1991, 3, 91.

16. Jasper, A.; Menegat, R.; Guerra-Sommer, M.; Cazzulo-Klepzig, M.; Souza, P. A.; J. South Am. Earth Sci. 2006, 21, 276.

17. Jasper, A.; Guerra-Sommer, M.; Uhl, D.; Salvi, J.; Kauffmann, M.; Osterkamp, I. C.; Gonçalves, C. V. Em Paleontologia: cenários de vida; Carvalho, I., Cassab, R. C. T., Schwanke, C., Carvalho, M. A., Fernandes, A. C. S., Rodrigues, M. A. C., Carvalho, M. S. S., Arai, M., Oliveira, M. E. Q., eds; Interciência: Rio de Janeiro, 2007.

18. Jasper, A.; Uhl, D.; Guerra-Sommer, M.; Mossbruger, V.; J. South Am. Earth Sci. 2008, 26, 4.

19. Jasper, A.; Guerra-Sommer, M.; Menegat, R.; Cazzulo-Klepzig, M.; Branco, F. S. R. T.; Salvi, J. Em Sítios Geológicos e Paleontológicos do Brasil; Winge, M., Schobbenhaus, C., Souza, C. R. G., Fernandes, A. C. S., Berbert-Born, M., Queiroz, E. T., eds.; 2009.

20. Jasper, A.; Guerra-Sommer, M.; Pesquisas 1998, 25, 1.

21. Costa, O. L.; Kionka, D. C. O.; Périco, E.; Jasper, A.; Geosul 2016, 31, 61.

22. Diniz, M. S.; Geo UFRJ 2001, 7.

23. Barbosa, L. C.; Espectroscopia no Infravermelho na Caracterização de Compostos Orgânicos, UFV: Viçosa, 2013.

24. Helfer, G. A.; Bock, F.; Mardera, L.; Furtado, J. C.; Costa, A. B.; Ferrão, M. F.; Quim. Nova 2015, 38, 4.

25. Padilla, E. R. D.; Nakashima, G. T.; Hansted, A. L. S.; Santos, L. R. O.; Barros, J. L.; Conti, A. C.; Yamaji, F. M.; Quim. Nova 2019, $42,5$.
26. Lara, D. M.; Tese de Doutorado, Universidade do Vale do Taquari, Brasil, 2017.

27. Balloni, C. J. V.; Trabalho de Graduação, Curso Engenharia Industrial Madeireira. UNESP, Brasil, 2009.

28. Yang, H.; Yan, R.; Chen, H.; Lee, D. H.; Zheng, C.; Fuel 2007, 86, 1781.

29. McKendry, P.; Biores. Technol. 2002, 83, 47.

30. Braadbaart, F.; Poole, I.; J. Archaeol. Sci. 2008, 35, 2434.

31. FAO Forestry Paper; Beaumont, E., ed.; 1985, p. 63.

32. Costa, L. J.; Dissertação de Mestrado, Universidade Federal de Lavras, Brasil, 2016.

33. Pereira, B. L. C.; Carneiro, A. C. O.; Carvalho, A. M. M. L.; Trugilho, P. F.; Melo, I. C. N. A.; Oliveira, A. C.; Rev. Arvore 2013, 37, 3.

34. Santos, R. C.; Carneiro, A. C. O.; Trugilho, P. F.; Mendes, L. M.; Carvalho, A. M. M. L.; Cerne 2012, 8, 1.

35. Figueroa, M. J. M.; Moraes, P. D.; Ambiente Construído 2009, 9, 4.

36. Guo, Y.; Bustin, R. M.; Int. J. Coal Geol. 1998, 37, 29.

37. Davrieux, F.; Rousset, P. L. A.; Pastore, T. C. M.; Macedo, L. A.; Quirino, W. F. Quim. Nova. 2010, 33, 5.

38. Klock, U.; Andrade, A. S. Química da madeira. eds., UFPR: Curitiba, 2013.

39. Lara, D. M.; Bresciani, L.; Osterkampc, I. C.; Hilgemannb, M.; Ethur, E.; Jasper, A. Ferrão, M. F.; Uhl, D.; Stülp, S. Quim. Nova. 2017, 40, 8.

40. Schmidt, I. D. Doutorado em Geociência. Universidade Federal do Rio Grande do Sul, Brasil, 2016.

41. Bica, J. B. Dissertação de Mestrado. Universidade do Vale do Taquari, Brasil, 2014. 